petrous segment of the ICA. Angiography and CTA confirmed pseudoaneurysm obliteration and complete vessel occlusion. Three-month follow-up CTA demonstrated stable vessel occlusion. Eleven months following ICA sacrifice, she started to complain of throat irritation and difficulty swallowing. During a scheduled sinus debridement, exposed coils were visualized in the right nasopharynx. CTA revealed vessel wall dehiscence with extrusion of coils into the nasopharynx but continued occlusion of the vessel. The exposed coils were clipped and extracted endoscopically for symptomatic relief. Three-month follow-up angiogram confirmed stable vessel occlusion and patient has remained asymptomatic.

Conclusion CBS can be a rare and devastating complication of head and neck surgery, often requiring immediate endovascular treatment. Coil migration is a rare complication following cerebral pseudoaneurysm obliteration via endovascular vessel sacrifice. When a patient has a partially extruding coil, it is imperative to obtain imaging and treat as soon as possible to avoid further coil migration and possible airway compromise.

Disclosures D. Sheinberg: None. D. McCarthy: None. E. Luther: None. S. Chen: None. R. Starke: None.

\section{E-084 RECONSTRUCTIVE AND DECONSTRUCTIVE ENDOVASCULAR TREATMENT OUTCOMES OF CAROTID BLOWOUT SYNDROME}

${ }^{1} \mathrm{~A}$ Sweidan*, ${ }^{2} \mathrm{~A}$ Schnure, ${ }^{2} \mathrm{Y}$ Yuki, ${ }^{1} \mathrm{R}$ Fujitani, ${ }^{2} \mathrm{~S}$ Suzuki. ${ }^{2}$ University of California, IIvine, Orange, $C A ;{ }^{2}$ Neurosurgery, University of California, Irvine, Orange, $C A$

\subsection{6/neurintsurg-2019-SNIS.159}

Introduction Carotid blowout syndrome (CBS) is a rare but dreaded complication of head and neck cancer with high mortality. Management of CBS requires a multidisciplinary approach; nevertheless, endovascular therapy remains the cornerstone in rendering hemostasis. Literature routinely describes successful hemostasis with either deconstructive (artery take down) or reconstructive (stenting or bypass) approach. Previous retrospective analysis suggests that outcomes are predicted by clinical severity at presentation, not treatment type. Methods Single-center, retrospective case review of endovascular treatment of CBS and outcome at University of California, Irvine.

Results Five cases of CBS were identified between 2012 to 2017. All patients were head and neck cancer treated with radiation. Of the five cases, one case failed to identify the source of bleeding which required open surgical ligation. In the remaining four patients, three achieved hemostasis with reconstruction, while the fourth was treated with deconstruction. In one of the three cases, reconstructive over deconstructive technique was chosen because the patient had bilateral lesions and previous embolization at the bleeding site failed to maintain hemostasis. All four patients were discharged from the hospital without any further complications related to the procedure achieving hemostasis. Perioperative mortality, stroke and infection rate was not identified. In the case of surgical ligation, the patient developed minor local site infection which was treated with antibiotics successfully.

Endovascular approaches permit evaluation of collateral circulation in situations where target artery warrants take down. Therefore, concurrent cerebral angiogram with endovascular therapy by way of stenting or sacrificing the target artery with embolic materials has become the alternative in otherwise inoperable condition as an emergent standard of care.

The figure shows an illustrative case of CBS with massive contrast extravasation (A), which was treated with a balloon mounted covered stent (B) due to bilateral lesions, and complete hemostasis was achieved (C).

Conclusion In our retrospective study we demonstrate continued endovascular therapy as a means of treatment for CBS to achieve emergent hemostasis. As previous literature describes successful hemostasis with either deconstructive or reconstructive technique; herein we describe four cases that required endovascular intervention with successful emergent hemostasis.

Disclosures A. Sweidan: None. A. Schnure: None. I. Yuki: None. R. Fujitani: None. S. Suzuki: None.

\section{E-085 CEREBRAL ISCHEMIC EVENTS AMONG PATIENTS UNDERGOING CAROTID ARTERY STENTING WITH OR WITHOUT EMBOLIC PROTECTION DEVICE-MULTICENTER REVIEW}

${ }^{1} \mathrm{Y}$ Lodi*, ${ }^{2} \mathrm{~V}$ Reddy, ${ }^{3} \mathrm{~S}$ Javed, ${ }^{4} \mathrm{~T}$ Wong, ${ }^{1} \mathrm{~S}$ Multani, ${ }^{5} \mathrm{~W}$ Elnour, ${ }^{6} \mathrm{~A}$ Harouni. ${ }^{1}$ Neurology, Neurosurgery and Radiology, Upstate Medical University, Binghamton/UHS-Wilson Medical Center, Johnson City, NY; ${ }^{2}$ Neurology, Neurosurgery and Radiology, UHS-Winson Medical Center/Upstate Medical University, Binghamton, NY, Johnson City, NY; ${ }^{3}$ Neurology, Neurosurgery and Radiology, UHS-Wilson Medical Center/Upstate Medical University, Binghamton, Johnson City, NY; ${ }^{4}$ Neurology and Neurosurgery, Maimanides Medical Centyer, Brooklyn, NY; ${ }^{5}$ Neurology and Neurosurgery, Upstate Medical University, Binghamton/UHS-Wilson Medical Center, Johnson City, NY; ${ }^{6}$ Department of Computer Science, Fort Hays State University, Hyas, KS

\subsection{6/neurintsurg-2019-SNIS.160}

Carotid artery stenting (CAS) with distal protection device (DPD) for stenosis $70 \%$ or higher is considered an
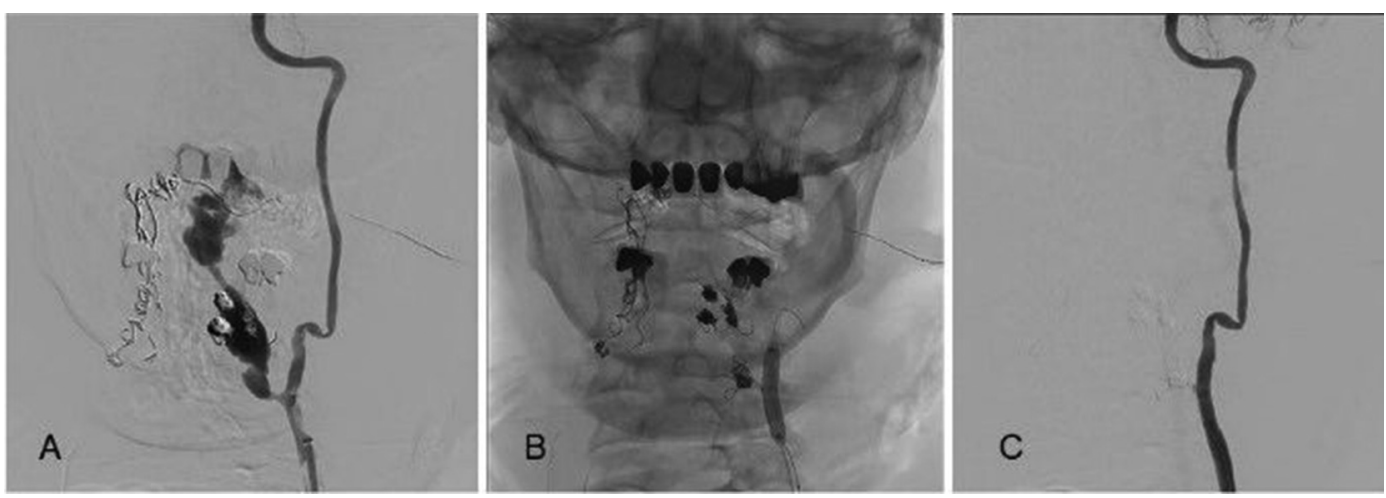
alternative to carotid artery endarterectomy (CEA) and approved by the central medical service (CMS) after the results of CREST trail. However, the incidence of cerebral ischemic event (CSE) was higher in CAS group compared to the CEA in CREST trial. DPD may not be utilized to patients who possess extreme tortuous vessels or near complete carotid artery stenosis, and many potential beneficiary CAS candidates are excluded. Objective of our study is to evaluate the incidence of CIE between groups who underwent CAS with or without the use of DPD. Additionally, we like to compare the overall perioperative complications between these two groups. Methods: From a prospectively maintained database, Consecutive patient who underwent CAS were collected and retrospectively analyzed from January 2008 to December 2018. Patients demographics, procedural techniques and perioperative events were captured. Outcome was measured using modified ranking scale (mRS) and NIHSS. Results: 165 consecutive patients (74\% symptomatic; stroke $35.25 \%$, TIA $38.85 \%$ ) with median age 69 (39-91) female 30.7\% underwent CAS (right ICA 57.55\%, left ICA 42.45\%) in two community based tertiary care facilities from 2008 to 2018. Median stenosis was $79 \%$ (70\%-99\%). DPD was used in $66 \%$ and $35 \%$ did not receive DPD due the anatomy. Demographic characteristics were similar between DPD (baseline mRS $0.33 \pm .67$ ) and non-DPD group $(0.59 \pm .15)$. The incidence of CIE was observed in 2 cases and both developed in DPD group; TIA in one and lacunar stroke NIHSS of 2, resolved in 90 days. Non-CIE related major complications was observed in two female patients; retroperitoneal hemorrhage related cardiac arrest leading to death in one in group without DPD and femoral angioseal related occlusion in one in DPD group who required repair and achieved mRS 0 in 90 days. Minor complications related to anterior small hematoma in 3 cases without impairing baseline $\mathrm{mRS}$ or hospital stay. In regards to the outcomes, 90 days mRS for DPD and non-DPD group was $0.49 \pm 1.1$ and $0.19 \pm 0.55$ respectively. Conclusions: Our study revealed no difference in the incidence of cerebral ischemic event in carotid artery stenting patients who were either treated with the use of DPD or without DPD. However, the overall incidence of CIE was low in our study and non-CIE related events were observed more in female patients. Larger CMS sponsored studies are required.

Disclosures Y. Lodi: None. V. Reddy: None. S. Javed: None. T. Wong: None. S. Multani: None. W. Elnour: None. A. Harouni: None.

\section{E-086 UNILATERAL CEREBRAL VASOCONSTRICTION FOLLOWING COMBINED CAROTID NEUROSURGICAL AND ENDOVASCULAR CAROTID REVASCULARIZATION}

L Sheikhi* ${ }^{*}$ J Tsai, M Bain, G Toth. Cleveland Clinic, Cleveland, $\mathrm{OH}$

\subsection{6/neurintsurg-2019-SNIS.161}

Introduction Reversible cerebral vasoconstriction syndrome (RCVS) can occur spontaneously or may be a secondary response. RCVS is rarely reported in literature following carotid revascularization and thought to be a possible result of chronic cerebral hypoperfusion with disturbance of cerebral autoregulation. There is no standard treatment for this condition. Here we report occurrence and management of cerebral vasoconstriction following unique combined neurosurgical and endovascular carotid revascularization for symptomatic carotid occlusion.

Methods We review a patient's clinical and imaging characteristics following carotid artery revascularization.

Results A female patient in her $70 \mathrm{~s}$ with history of hypertension, controlled type 2 diabetes mellitus, and current smoker presented with recurrent episodes of transient left sided limb shaking. She was found to have a right internal carotid artery (ICA) occlusion at the cervical origin with a non-flow limiting $40 \%$ stenosis of the left ICA associated with an acute infarct in the right frontal white matter. A diagnostic cerebral angiogram confirmed the occlusion of the right ICA with limited and delayed retrograde filling from the vertebrobasilar system via the right posterior communicating artery and smaller collateral contribution via right external carotid to ophthalmic artery. Notably, there was no anterior communicating artery for contralateral cross-filling. She had several outpatient visits and hospital admissions for hypotensive episodes associated with recurrent stereotypic symptoms. Repeat imaging demonstrated overall small stroke burden and no hemorrhage. Upon careful discussion and review of possible options, a combined neurosurgical-endovascular carotid revascularization was performed with endarterectomy of an occlusive plaque involving the distal common carotid to the proximal ICA, and direct mechanical thrombectomy with aspiration and stentriever of the cervical ICA to the terminus. Additional angioplasty and stenting of proximal petrous ICA segment was performed via femoral access after closure of the surgical site. She clinically was doing well until 5 days post-operatively, when she developed recurrent symptoms. Imaging showed small new ischemic strokes without hemorrhage. Continuous video-EEG
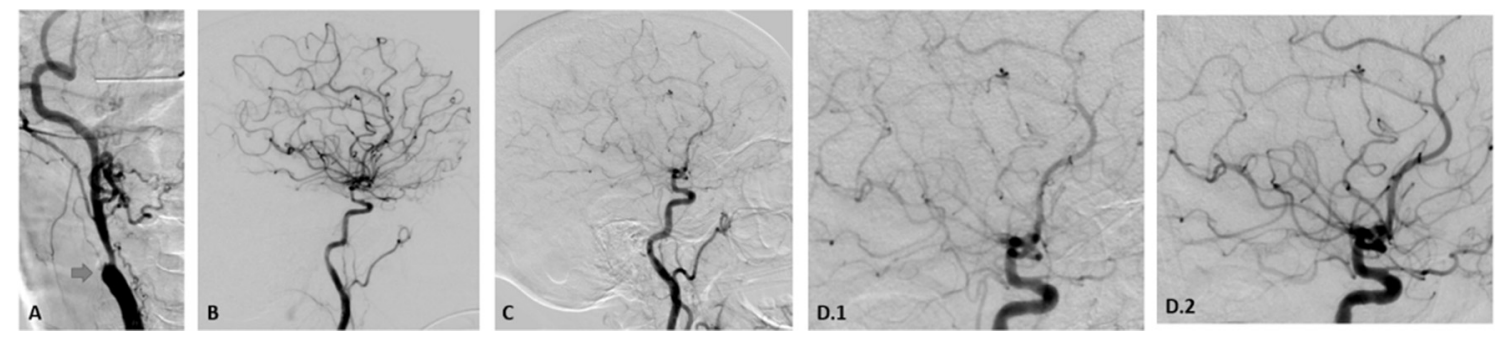

Abstract E-086 Figure 1 Initial and interval cerebral angiograms of right internal carotid artery (ICA) Figure A is an anterior view of the abrupt right cervical ICA occlusion at the bifurcation (arrow). Figure B demonstrates robust right ICA filling upon final review of combined neurosurgicalendovascular revascularization of the right ICA (lateral view). Following symptom recurrence, Figure $C$ is a repeat angiogram demonstrating interval right ICA focal severe segmental irregularities consistent with cerebral vasoconstriction. Figure D.1 is a magnified view of Figure B, followed 10 minutes post intra-arterial verapamil treatment with improvement in vessel caliber on Figure D.2. 